\title{
Transcatheter heart-valve replacement: update
}

\author{
Michael W.A. Chu MD MEd, Michael A. Borger MD PhD, Friedrich W. Mohr MD PhD, \\ Thomas Walther MD PhD
}

Previously published at www.cmaj.ca

A ortic stenosis is a common heart disease that results in a fixed obstruction of the left ventricle and presents with angina, syncope, congestive heart failure or sudden death. About 3\% of the general population over 75 years of age have severe aortic stenosis, ${ }^{1}$ and $2 \%$ of the general population at large have bicuspid aortic valves. Valve replacement remains the only definitive therapy for patients with severe aortic stenosis. ${ }^{2}$ However, large numbers of high-risk patients are never referred or conventional valve surgery is deemed too risky for them. As a result, many high-risk patients are managed conservatively, ${ }^{3,4}$ despite the poor prognosis associated with medical therapy. ${ }^{5}$

Innovative techniques and emerging technologies have been focused on such high-risk patients, enabling less invasive valve replacement to be performed without the need for sternotomy, use of heart-lung bypass machines or even stopping the heart. Avoidance of these conventional techniques may be prudent because of their known risks and deleterious effects. ${ }^{4}$

Stent-based transcatheter valve replacement now offers patients a less invasive alternative with potentially reduced risks, which may be particularly beneficial for elderly, high-risk patients. Investigations into transcatheter approaches to heartvalve repair, particularly for mitral insufficiency, are currently underway. Our review focuses on the clinical results and future possibilities for transcatheter valve replacement therapy.

\section{Transcatheter valve design}

The principles of this technology are aimed at relieving the stenosis of the native valve and creating a functioning valve that can be delivered via a catheter-based system on the beating heart, without the need for a heart-lung machine or sternotomy. The design and construction of the device itself consists of xenogenic pericardial cusps mounted within an expandable stent that relies upon the forces of radial traction to seat the prosthesis tightly within the native aortic annulus. A tight seal within the cusps of the crushed native aortic valve and the surrounding annulus is necessary to prevent paravalvular leakage.

Transcatheter valves can be implanted within the native aortic valve via the femoral artery, the left ventricular apex or, less commonly, the axillary artery. Guidewires and sheaths are placed through these vascular access sites to reach the level of the aortic valve, where a balloon valvuloplasty is performed to relieve the stenosis. Then, the prosthesis is guided up to the level of the aortic valve and implanted under rapid

\section{Key points}

- Transcatheter valve replacement offers patients less invasive heart-valve therapy, with lower morbidity and mortality, and quicker recovery. A dedicated team is essential for the success of this program.

- The procedure can be performed for the aortic and pulmonic valves.

- Minimally invasive techniques enable valve implantation, as well as repeat valve replacement, without the need for cardiopulmonary bypass or sternotomy.

ventricular pacing to temporarily decrease the cardiac output. Congenital pulmonic valve stenosis can also be addressed in a similar fashion via the femoral vein.

Currently, three products are commercially available, all of which have received European conformity (i.e., CE mark) approval: the SAPIEN (Edwards Lifesciences) and CoreValve (Medtronic) devices, which are for aortic valve replacement, and the Melody (Medtronic) device which is for pulmonic valve replacement in congenital heart disease (Figure 1).

\section{Patient selection}

Initially, patients were selected for transcatheter aortic valve replacement on a compassionate basis only, such that the valves were applied to moribund patients without traditional surgical options. As the feasibility, safety and efficacy of these procedures gradually become established, patients with severe, symptomatic aortic stenosis who are of advanced age and have high-risk profiles are being selected for these techniques. Advanced age is generally considered to be 75 years or older, although lower age cutoffs have been used in some institutions.

Surgical risk is generally assessed using a combination of scoring systems (e.g., a EuroSCORE [www.euroscore.org] greater than 9 points, a Society of Thoracic Surgeons [http://209.220.160.181/STSWebRiskCalc261/] score greater than $10 \%$ ) and clinical judgement. Generally, a predicted mortality risk of greater than $15 \%$ with conventional aortic valve

From the Division of Cardiac Surgery (Chu), Department of Surgery, Lawson Health Research Institute, University of Western Ontario, London, Ont.; and Klinik für Herzchirurgie (Chu, Borger, Mohr, Walther), Herzzentrum Leipzig, Universität Leipzig, Leipzig, Germany

CMAJ 2010. DOI:10.1503/cmaj.080064 
replacement is considered high risk. ${ }^{6}$ Certain conditions, such as a highly calcified (i.e., "porcelain") aorta, mediastinal irradiation, liver cirrhosis or a need for reoperation with patent bypass grafts, may lower the threshold of risk for transcatheter valves, because of the well-recognized increased risks associated with these conditions in conventional surgery.

Transcatheter therapy is usually not offered to patients with asymptomatic aortic stenosis or patients with a life expectancy of less than one year. Although most patients will have a suitably sized aortic annulus (as assessed by echocardiography), patients with a large aortic annulus are not currently candidates for transcatheter aortic valve implantation because of a limited selection of prosthetic sizes. Our current indications and contraindications for transcatheter aortic valve implantation are detailed further in Box 1. An ongoing randomized clinical trial in Europe and North America is expected to further delineate the optimal patient groups for transfemoral and transapical aortic valve therapy.

Transcatheter pulmonic valve replacement is indicated in patients with congenital, symptomatic pulmonary valve stenosis or insufficiency who have previously undergone right ventricular outflow tract reconstruction, usually for tetralogy of Fallot. The most common indication is homograft stenosis with calcification. Most patients are young (in contrast to transcatheter aortic valve patients), but have undergone multiple previous operations and are therefore deemed to be at increased risk with conventional surgery.

\section{Preoperative workup}

A history and physical examination, as well as routine preoperative blood work, 12-lead electrocardiography, and chest radiography, are performed initially to assess the patient's candidacy for transcatheter valve therapy. Transthoracic echocardiography defines the character and severity of the aortic stenosis. However, transesophageal echocardiography is often necessary to better delineate the calcification pattern and accurately measure the aortic annulus and root diameters. Currently, limitation in the sizes of prostheses require patients to have a specific aortic annulus diameter for proper stent deployment and seating (Box 1). To decrease the risk of paravalvular leak, the implanted prosthesis is slightly oversized. Because of the advanced age of the patients, coronary angiography is performed. Computed tomography is also recommended for all patients to define the distances between the aortic annulus and the coronary ostia, to minimize risks of coronary occlusion.

\section{Techniques used in valve implantation}

Transcatheter valve implantations are best performed by a technologically adept team of cardiac surgeons, cardiologists and anesthetists. Recommended training often consists of a combination of didactic learning, hands-on simulation, and proctorship by experts in the area. Although these procedures can be performed in cardiac catheterization suites, a recent expert panel suggested that they are best performed in a hybrid operating theatre with fixed fluoroscopic imaging. ${ }^{9}$ A hybrid operating theatre ensures sterility and the ability to rapidly convert to a conventional surgical approach during unforeseen complications, while maintaining the fluoroscopic capabilities of a catheterization laboratory.

The benefit of a catheter-based approach is that it is less invasive, especially in avoidance of use of a heart-lung machine and sternotomy. However, a "safety net" heart-lung bypass circuit is primed and available if urgently needed.

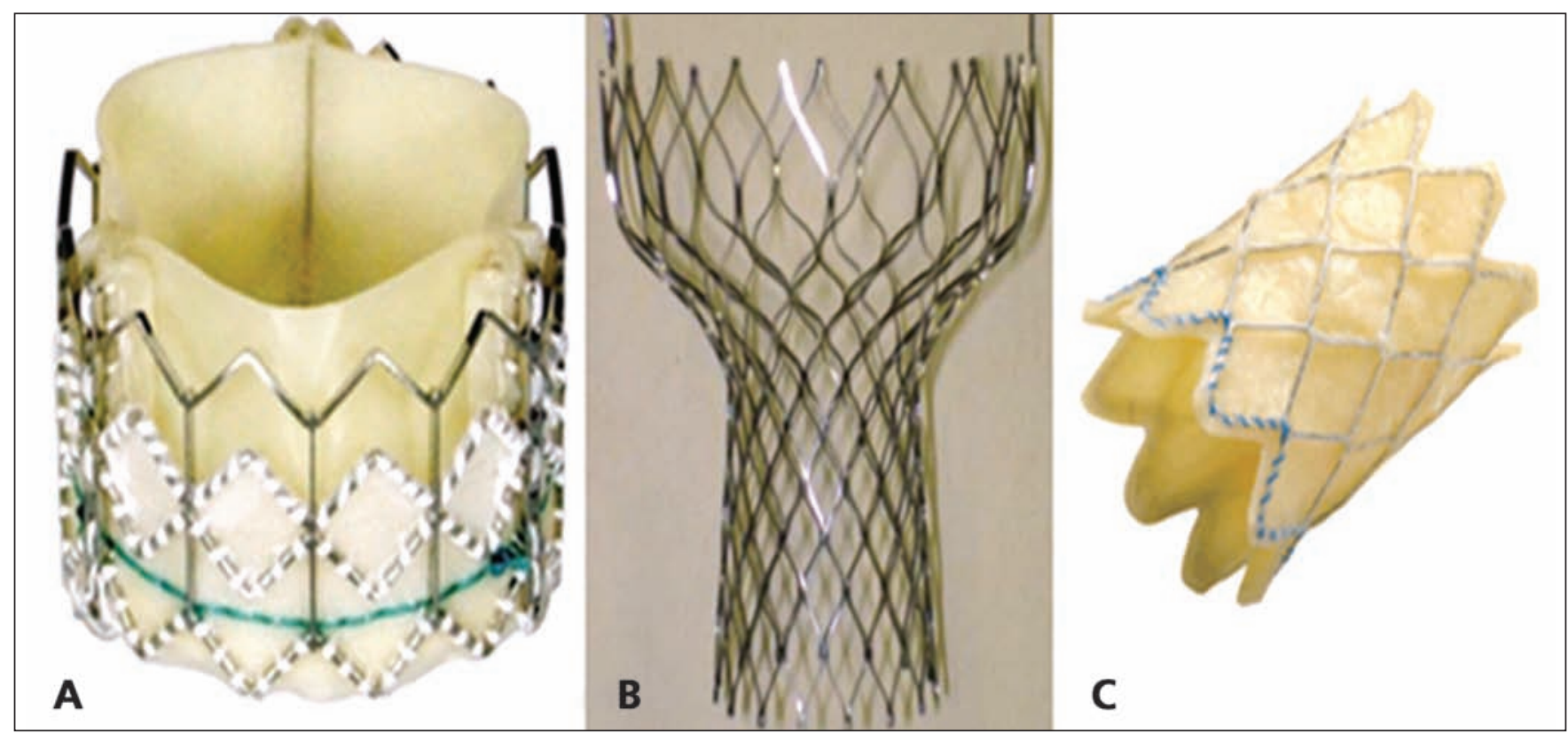

Figure 1: (A) The balloon-expandable SAPIEN (Edwards Lifesciences) transcatheter prosthesis for aortic valve implantation. (B) The selfexpanding CoreValve (Medtronic) transcatheter prosthesis stent for aortic valve implantation. (C) The balloon-expandable Melody (Medtronic) prosthesis for pulmonic valve implantation in congenital heart disease. 
Box 1: Indications and contraindications for transcatheter aortic valve implantation ${ }^{6-8}$

Indications

- Severe aortic stenosis

- Patient age of more than 75 years

- Significant comorbidities

- Estimated operative risk greater than $15 \%$

- EuroSCORE greater than 9 points (logistic EuroSCORE greater than $15 \%$ )

- Society of Thoracic Surgeons score greater than $10 \%$

- Aortic annulus diameter of 18-24-mm (SAPIEN, Edwards Lifesciences)

- Aortic annulus diameter of 20-27-mm (CoreValve, Medtronic)

- Specific conditions that may benefit from a transcatheter approach

- Highly calcified aorta

- Mediastinal irradiation

- Previous coronary bypass surgery with patent bypass grafts

\section{Contraindications}

- Low risk for conventional aortic valve replacement

- Isolated aortic insufficiency

- Need for concomitant coronary or other valve operation

- Ascending aortic aneurysm

- Aortic annulus diameter of less than $18 \mathrm{~mm}$ or greater than $27 \mathrm{~mm}$

- Life expectancy of less than 12 months

Access to the aortic valve is achieved with guidewires, regardless of the approach. The transfemoral technique requires adequately sized (i.e., greater than $7 \mathrm{~mm}$ in diameter), nontortuous femoral and iliac access vessels, along with a relative paucity of aortic atherosclerosis. Presence of any of these factors would favour a transapical approach. The transapical approach is advantageous because it does not have any limitations with regard to access vessel size, requiring a small 5-7 cm anterolateral thoracotomy incision over the left ventricular apex. After balloon valvuloplasty, the tran- scatheter valve prosthesis is brought to the level of the native aortic valve and deployed in a sequential stepwise manner (Figure 2).

Transcatheter pulmonary valve replacement can be performed with a low risk of sudden hemodynamic deterioration because of the favourable anatomy and hemodynamics of the right heart. These procedures are therefore usually performed in the cardiac catheterization laboratory, and occasionally under local anesthetic only. Access to the right ventricular outflow tract is gained via the femoral vein, which has fewer size restrictions than the femoral artery. In general, all of the above transcatheter procedures can be accomplished in one to two hours.

\section{Postoperative recovery}

Most patients can expect a short stay in a postanesthetic care unit, where they recover from the anesthesia and are subsequently transferred to a step-down unit. Monitoring and treatment in an intensive care unit is not necessary for most patients. All invasive monitoring lines and chest tubes are removed within 24 hours of surgery if no complications develop. Cardiac arrhythmia monitoring is essential because of the risk of heart block in 5\%-10\% of patients when calcium is compressed against the conduction tissues. Although the degree of surgical trauma is less than that with conventional surgery, many of these patients require prolonged hospital stays because of substantial comorbidities. Patients with transcatheter valves are usually discharged on an antiplatelet regimen, consisting of acetylsalicylic acid with or without clopidogrel. Unlike sternotomy patients, who require three months of precautions for adequate sternal healing, transcatheter valve patients experience a much quicker recovery with good wound healing and a return to regular activities two to three weeks after discharge.

\section{Possible complications}

Possible complications can be divided into early and late. Early perioperative complications include stent malpositioning (about $10 \%$ ), stent migration (less than $5 \%$ ), coronary obstruction

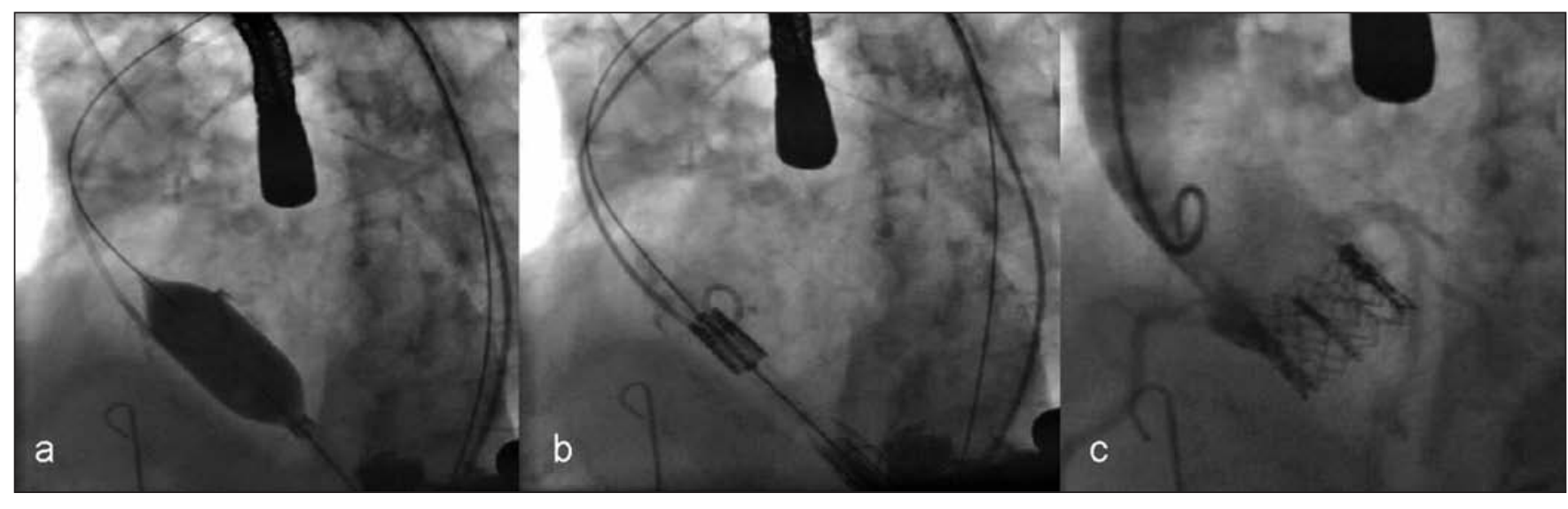

Figure 2: (A) Balloon aortic valvuloplasty. (B) Positioning of the undeployed transcatheter valve prosthesis within the aortic annulus. (C) Intraoperative aortography confirming good implantation of the device, without coronary obstruction or paravalvular leak. 
(about 5\%) and substantial paravalvular leak (about 10\%).,10-15 Problems related to vascular access, including iliac rupture and peripheral leg ischemia, can occur with the transfemoral technique, whereas left ventricular bleeding can occur with use of the transapical technique. Safety "bailout" measures involving emergency heart-lung bypass can help avoid hemodynamic collapse in instances of catastrophic complications. Percutaneous stenting of partially obstructed coronary arteries, or bypass grafting of completely obstructed coronary arteries, can be performed during heart-lung machine support. Small degrees of paravalvular leak are common, and can occur in up to $50 \%$ of patients. However, these are usually well tolerated and rarely clinically significant. Hemolysis has not yet been reported as a complication of these devices.

Late complications are usually related to comorbidities of the patient, such as pulmonary or renal insufficiency. Although generally uncommon, stroke may occur with a higher frequency during transfemoral implantations (Table 1), ${ }^{6,10-15}$ and is likely related to the increased aortic arch manipulations when compared with the transapical approach. Since the advent of this technology is so recent, little data exists on additional late-term complications. However, patients should be monitored for the development of late heart block, endocarditis and signs of valve dysfunction with worsening aortic insufficiency and congestive heart failure. Patients with transcatheter valves should undergo endocarditis prophylaxis for dental work and minor procedures.

\section{Early results}

Initial results have been encouraging, and a few centres have developed enough experience to establish the safety and efficacy of these devices. The mean patient age in all series was early in the ninth decade of life, and most patients had significant comorbidities. All case series have shown significant improvements in the aortic valve area and significant reduction in transvalvular gradients from pre- to post-procedure (Table 1). ${ }^{6,10-15}$ In general, the early 30 -day procedural mortal- ity has been reported to be $10 \%-12 \%$ for larger case series involving both transfemoral and transapical AVR approaches, ${ }^{12-14}$ and one-year survival has been around $80 \%{ }^{12,13,15}$ Historically, most of these patients would have been considered too high-risk for conventional surgery; medical management alone has a poor prognosis, with one-year survival recently reported to be about $50 \% .^{16,17}$

Rates of clinical failure have been $5 \%-10 \%$. Procedural success is slightly higher in the transapical group, likely because of difficulties related to peripheral access in the transfemoral group. Postprocedural morbidity is similar between the two techniques, with the exception of stroke and neurologic injury which have been reported in some series to occur at a higher incidence in the transfemoral approach with both the SAPIEN and CoreValve prostheses ${ }^{12-15}$ (Table 1).

Published results have included mostly small case series from centres of expertise in Canada and Europe, and involve 50 or fewer patients each. ${ }^{6.13,14,18,19} \mathrm{In}$ addition, a few nonrandomized, multicentre reports have collated data from experienced centres with the early results of some developing centres. ${ }^{12,15,20}$ Each series reports centre-specific preferences for transcatheter devices and routes for implantation. Currently, no good evidence exists to evaluate either the optimal transcatheter valve device, the optimal approach to transcatheter valve implantation, or whether or not transcatheter valve implantation prolongs survival as compared with medical therapy in these high-risk patient populations. Although these small series have certainly demonstrated proof of concept, these results must be carefully interpreted because the patients involved are higher risk and the total numbers involved are small — hence magnifying the effect of each complication at this early stage in the learning curve.

The limitations of such studies also include important potential biases, given that the results are derived from centres with specified equipment, organized multidisciplinary teams, specialized skills and interests, and unique referral patterns - characteristics that allow for stakeholder development within the new transcatheter field. As with any new

Table 1: Summary of published results of transcatheter aortic valve implantation

\begin{tabular}{|c|c|c|c|c|c|c|c|c|c|c|c|}
\hline \multirow[b]{2}{*}{ Study } & \multirow[b]{2}{*}{ Access } & \multirow[b]{2}{*}{ Device } & \multirow{2}{*}{$\begin{array}{l}\text { No. of } \\
\text { patients }\end{array}$} & \multirow{2}{*}{ Study design } & \multirow{2}{*}{$\begin{array}{c}\text { Logistic } \\
\text { EuroSCORE, \% }\end{array}$} & \multirow{2}{*}{$\begin{array}{l}\text { 30-day } \\
\text { mortality, \% }\end{array}$} & \multirow[b]{2}{*}{ Stroke, \% } & \multicolumn{2}{|c|}{$\begin{array}{l}\text { Aortic valve area, } \\
\mathrm{cm}^{2} \text { (SD) }\end{array}$} & \multicolumn{2}{|c|}{$\begin{array}{l}\text { Mean transvalvular } \\
\text { gradient, } \mathrm{mmHg} \text { (SD) }\end{array}$} \\
\hline & & & & & & & & Before & After & Before & After \\
\hline $\begin{array}{l}\text { Cribier et al., }{ }^{10} \\
2006\end{array}$ & Transfemoral & $\begin{array}{l}\text { Cribier- } \\
\text { Edwards }\end{array}$ & 27 & Single centre & $\sim 27$ & 22.0 & 4.0 & $0.6(0.1)$ & $1.7(0.1)$ & $37(13.0)$ & $9(2.0)$ \\
\hline $\begin{array}{l}\text { Grube et al.., } \\
2006\end{array}$ & Transfemoral & CoreValve & 25 & Single centre & 11 & 20.0 & 4.0 & $0.7(0.1)$ & NR & $44(11.0)$ & $12(3.0)$ \\
\hline $\begin{array}{l}\text { Lichtenstein et } \\
\text { al., }{ }^{11} 2006\end{array}$ & Transapical & $\begin{array}{l}\text { Cribier- } \\
\text { Edwards }\end{array}$ & 7 & Single centre & 35 & 14.0 & None & $0.7(0.1)$ & $1.8(0.8)$ & $31(10.0)$ & $9(6.0)$ \\
\hline $\begin{array}{l}\text { Grube et al.., } \\
2007\end{array}$ & Transfemoral & CoreValve & 86 & Multicentre & 22 & 12.0 & 10.0 & $0.6(0.2)$ & NR & $44(15.0)$ & NR \\
\hline $\begin{array}{l}\text { Webb et al.., } \\
2007\end{array}$ & Transfemoral & SAPIEN & 50 & Single centre & 28 & 12.0 & 4.0 & $0.6(0.2)$ & $1.7(0.4)$ & $46(17.0)$ & $11(5.0)$ \\
\hline $\begin{array}{l}\text { Walther et al., } \\
2007\end{array}$ & Transapical & SAPIEN & 30 & Single centre & 27 & 7.0 & None & NR & NR & $43(14.0)$ & $8(5.0)$ \\
\hline $\begin{array}{l}\text { Walther et al., }{ }^{15} \\
2007\end{array}$ & Transapical & SAPIEN & 59 & Multicentre & 27 & 13.6 & 3.4 & $0.5(0.2)$ & NR & NR & $9(6.0)$ \\
\hline
\end{tabular}

Note: $\mathrm{NR}=$ not reported, $\mathrm{SD}=$ standard deviation. 
technology and developing field, industry plays an important supportive role that must be held within the confines of appropriate ethical and research-related behaviour. Scientific rigour must be applied to determine the future application of transcatheter valve technology. A randomized clinical trial evaluating the SAPIEN device is underway in Europe and North America to assess efficacy and one-year mortality.

Studies are underway to evaluate the longer-term efficacy of transcatheter valves. In-vitro studies suggest that the longterm durability of these valves may be comparable to traditional valve-replacement prostheses, but careful follow-up will be required. Although transcatheter valves have many components (e.g., pericardial leaflets, anticalcification treatment) that exist in conventional xenograft valves with proven long-term results, it remains to be seen whether valve crimping will have any impact on long-term function. The issue of long-term durability will become paramount when younger patients are considered for transcatheter valve replacement a development that may become inevitable as experience develops, early complication rates improve, and general enthusiasm grows for these innovative techniques.

\section{Recent advances and future directions}

Recently, transcatheter valves were also shown to be appropriate for replacement of previously implanted conventional bioprostheses that have undergone structural valve deterioration in either the aortic or mitral position. ${ }^{21,22}$ The transcatheter valve is simply deployed within the stent of the previously implanted conventional valve, creating a valve-in-a-valve configuration. This technique may offer patients the possibility of a much less invasive second- or third-time aortic valve reoperation, with potentially less morbidity and quicker recovery.

Future development of transcatheter valves must focus on improvements in design that facilitate easy and accurate positioning, with avoidance of coronary obstruction and minimization of paravalvular leak. In addition, the ability to retrieve and redeploy a malpositioned valve would be an invaluable capability that is currently lacking in all devices. Future research should also focus on miniaturization of delivery platforms, as this will always represent a technological advancement and will increase the number of patients who are potential candidates. Improvements in intraoperative fluoroscopic, echocardiographic and possibly magnetic resonance imaging will also likely improve the success of these techniques. Further clinical research should focus on which patients are served best by which device and which technique, whether it be transfemoral, transapical or conventional surgery.

This article has been peer reviewed.

Competing interests: Thomas Walther has received speaker fees from Edwards Lifesciences. None declared for Michael Chu, Michael Borger and Friedrich Mohr.

Contributors: Michael Chu, Michael Borger and Thomas Walther were involved in the conception of the manuscript, and all of the authors were involved in the development of intellectual content. Michael Chu drafted the manuscript. All of the authors critically reviewed the manuscript and approved the final version submitted for publication.

\section{REFERENCES}

1. Lindroos M, Kupari M, Heikkila J, et al. Prevalence of aortic valve abnormalities in the elderly: an echocardiographic study of a random population sample. $J \mathrm{Am}$ Coll Cardiol 1993;21:1220-5.

2. Bonow RO, Carabello BA, Kanu C, et al. ACC/AHA 2006 guidelines for the management of patients with valvular heart disease: a report of the American College of Cardiology/American Heart Association Task Force on Practice Guidelines. Circulation 2006;114:e84-231.

3. Iung B, Baron G, Butchart EG, et al. A prospective survey of patients with valvular heart disease in Europe: the Euro heart survey on valvular heart disease. Eur Heart $J$ 2003;24:1231-43.

4. Varadarajan P, Kapoor N, Bansal RC, et al. Clinical profile and natural history of 453 nonsurgically managed patients with severe aortic stenosis. Ann Thorac Surg 2006;82:2111-5

5. Elahi MM, Khan JS, Matata BM. Deleterious effects of cardiopulmonary bypass in coronary artery surgery and scientific interpretation of off-pump's logic. Acute Card Care 2006;8:196-209.

6. Grube E, Laborde JC, Gerckens U, et al. Percutaneous implantation of the CoreValve self-expanding valve prosthesis in high-risk patients with aortic valve disease: the Siegburg first-in-man study. Circulation 2006;114:1616-24.

7. The PARTNER trial. Placement of AoRTic TraNscathetER valve trial. ClinicalTrials, gov ID\#: NCT00530894. Available: http://clinicaltrials.gov/ct2 /show/NCT00530894 (accessed 2009 Dec. 14).

8. Walther T, Dewey T, Borger MA, et al. Transapical aortic valve implantation: step by step. Ann Thorac Surg 2009;87:276-83.

9. Rosengart TK, Feldman T, Borger MA, et al. Percutaneous and minimally invasive valve procedures: a scientific statement from the American Heart Association Council on Cardiovascular Surgery and Anesthesia, Council on Clinical Cardiology, Functional Genomics and Translational Biology Interdisciplinary Working Group, and Quality of Care and Outcomes Research Interdisciplinary Working Group. Circulation 2008;117:1750-67.

10. Cribier A, Eltchaninoff $\mathrm{H}$, Tron $\mathrm{C}$, et al. Treatment of calcific aortic stenosis with the percutaneous heart valve: mid-term follow-up from the initial feasibility studies: the French experience. J Am Coll Cardiol 2006;47:1214-23.

11. Lichtenstein SV, Cheung A, Ye J, et al. Transapical transcatheter aortic valve implantation in humans: initial clinical experience. Circulation 2006;114:591-6.

12. Grube E, Schuler G, Buellesfeld L, et al. Percutaneous aortic valve replacement for severe aortic stenosis in high-risk patients using the second- and current third-generation self-expanding CoreValve prosthesis: device success and 30-day clinical outcome. J Am Coll Cardiol 2007;50:69-76.

13. Webb JG, Pasupati S, Humphries K, et al. Percutaneous transarterial aortic valve replacement in selected high-risk patients with aortic stenosis. Circulation 2007;116:755-63.

14. Walther T, Falk V, Borger MA, et al. Minimally invasive transapical beating heart aortic valve implantation - proof of concept. Eur J Cardiothorac Surg 2007;31:9-15.

15. Walther T, Simon P, Dewey T, et al. Transapical minimally invasive aortic valve implantation: multicenter experience. Circulation 2007;116:I240-5.

16. Kapadia SR, Goel SS, Svensson L, et al. Characterization and outcome of patients with severe symptomatic aortic stenosis referred for percutaneous aortic valve replacement. J Thorac Cardiovasc Surg 2009;137:1430-5.

17. Dewey TM, Brown DL, Das TS, et al. High-risk patients referred for transcatheter aortic valve implantation: Management and outcomes. Ann Thorac Surg 2008;86:1450-7.

18. Walther T, Falk V, Kempfert J, et al. Transapical minimally invasive aortic valve implantation; the initial 50 patients. Eur J Cardiothorac Surg 2008;33:983-8

19. Ye J, Cheung A, Lichtenstein SV, et al. Six-month outcome of transapical transcatheter aortic valve implantation in the initial seven patients. Eur J Cardiothorac Surg 2007;31:16-21.

20. Svensson LG, Dewey T, Kapadia S, et al. United States feasibility study of transcatheter insertion of a stented aortic valve by the left ventricular apex. Ann Thorac Surg 2008;86:46-54; discussion 5 .

21. Walther T, Falk V, Dewey T, et al. Valve-in-a-valve concept for transcatheter minimally invasive repeat xenograft implantation. J Am Coll Cardiol 2007;50:56-60.

22. Walther T, Kempfert J, Borger MA, et al. Human minimally invasive off-pump valve-in-a-valve implantation. Ann Thorac Surg 2008;85:1072-3.

Correspondence to: Dr. Michael W.A. Chu, Division of Cardiac Surgery, Rm. B6-106, University Hospital, London Health Sciences Centre, 339 Windermere Rd., London ON N6A 5A5; michael.chu@lhsc.on.ca

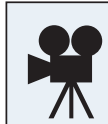
See video online: Transcatheter aortic valve replacment http://www.cmaj.ca/video/trans-chu-v1.wmv 